\title{
Ensuring timetable stability with train traffic data
}

\author{
T. Graffagnino \\ SBB AG, Switzerland
}

\begin{abstract}
Swiss Federal Railways plan and operate one of the densest rail networks in the world. Over the last 8 years, punctuality on the network has been steadily increasing. Figures comparing train traffic density and showing the punctuality of Swiss trains over the last 8 years will be presented and briefly discussed. Then the process of collecting the train traffic data at SBB will be explained and discussed.

The main points of this paper will be the mathematical methods and the graphical data visualisation method developed by SBB to continually optimise the timetable with extensive use of historical train traffic data.

Based on quite simple collected data - actual arrival and arrival delay, actual departure and departure delay - for each train at about 1000 stations, the paper will define and review the different types of analysis that the method facilities: arrival punctuality, departure punctuality, dwelling time, journey time, dwelling time deviation and journey time deviation.

The paper will also discuss the type of distribution function which can be observed and explain why the original statistical definition of boxplots by John Wilder Tukey (Exploratory Data Analysis, Addison-Wesley) has been adapted for rail-related use. Subsequently, the strength of this new defined boxplot is demonstrated with a top-down delay cause analysis starting with punctuality in Switzerland, then comparing the major Swiss stations and the train family, and finally identifying the problems on the corridors. We then show how the usage of median insights into the dynamics of the railway system helps us to identify potential stability problems.

To conclude, the identification and visualisation of knock-on delay between trains based on actual train traffic data is addressed as an interesting open issue. Keywords: timetable stability, traffic data, statistic, boxplot, punctuality, cyclic timetable, data mining.
\end{abstract}




\section{Introduction}

Since 2003, the density of rail traffic in Switzerland has increased with each new timetable:

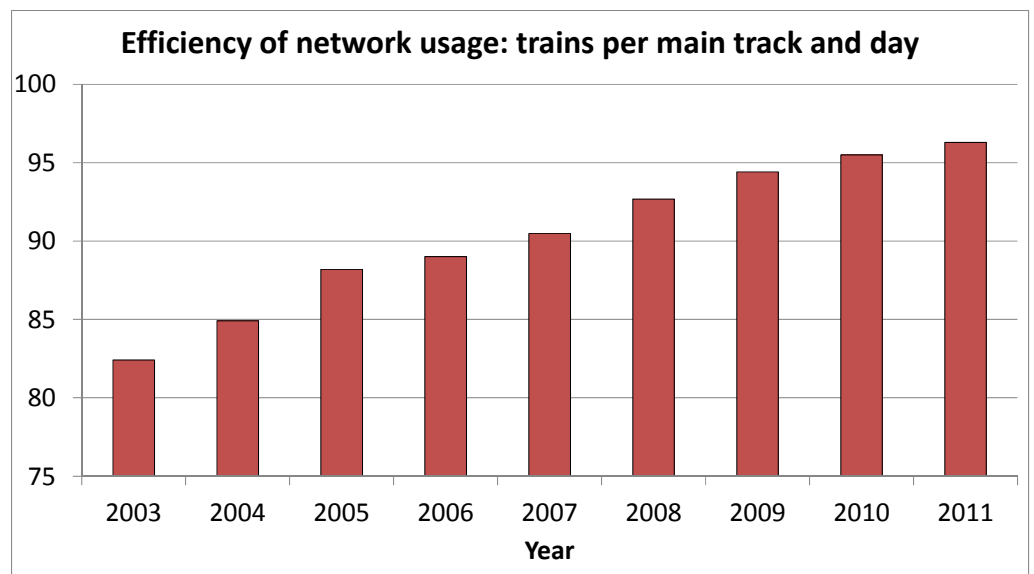

Figure 1: $\quad$ Number of trains per main track and day.

In parallel with this increase in traffic density, SBB has succeeded in improving its punctuality:

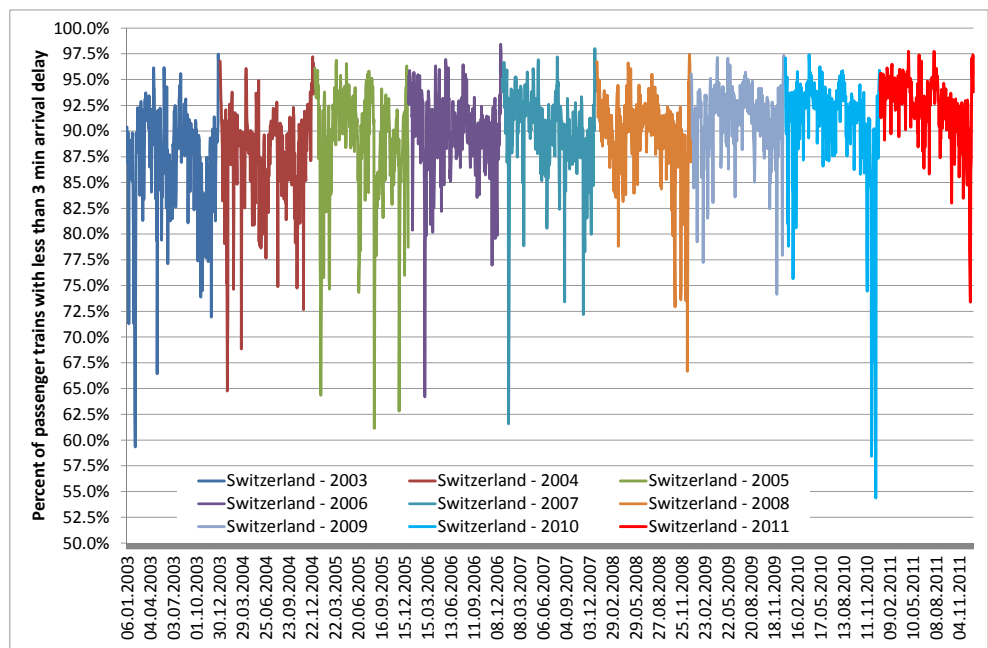

Figure 2: $\quad$ Daily 3-minute punctuality Monday to Friday 2003 to 2011.

The 3-minute arrival punctuality of passenger trains has improved considerably since 2003. A seasonal effect is evident; punctuality deteriorates 
each year during the autumn and winter periods. The cold spell at the end of 2010 also had a noticeable impact, with punctuality falling to its lowest levels since 2003.

Numerous measures were taken in order to achieve these positive results in terms of traffic density and punctuality. Timetable schedulers carried out finetuning and continual adjustments to reserve times. This was made possible by regular analysis of train movement data. We shall now present the latest developments that have been implemented in this area, as well as some actual cases and information on how to interpret the graphs.

\section{Train traffic data mining}

At SBB, railway tracks are fitted with track circuits or axle counters. This equipment is linked to interlocking systems which are themselves operated by remote control. As a train moves around the network it is continuously monitored by remote control via the occupancy reports that are logically linked to its train number. Each time the train passes a main signal, it will occupy a new section and this event will be picked up by the remote control. The event will be simultaneously transmitted to the "LTA" (Leittechnik Adapter) system, which records the date and the exact time to the second. This information is constantly being collected by the "RCS" (Rail Control System) traffic monitoring system and displayed in real time so that any problems to do with train movements can be identified and resolved. During the night, all the data collected by the RCS during the day is archived in a database and made available to the analysis tools.

In order to be able to check whether trains are keeping to the timetable at each stopping point, the RCS system extrapolates from the train movements between the signal and the stopping point. To extrapolate the time of stopping (or departure), the RCS system adds (or subtracts) the appropriate journey time to (or from) the time at which the train passed the signal. This journey time is calculated in real time with reference to the routes being used and the exact composition of the train. The quality of this extrapolation is determined by the variability of the driving behaviours observed on the ground. With the help of a system fitted to SBB's regional trains, we have been able to compare the data retrieved from the RCS system with the actual moment the train stopped, as sent by the on-board GPS:

This graph shows that for 12 of the 13 key nodes, the median error is less than or equal to 10 seconds. The interquartile range is less than 18 seconds for 12 nodes. The two nodes OL (Olten) and LS (Lausanne) with an interquartile range of 24 seconds for OL and a median error of 18 seconds for LS, are the ones with the largest error; this is principally owing to the very different driving behaviours at these nodes. It can be seen that the aberrant figures on this graph are systematically negative. This can be explained by the fact that the data is collected at the entrance signal, so any slower speed, for example due to a warning signal, will have the effect of increasing the real journey time. 


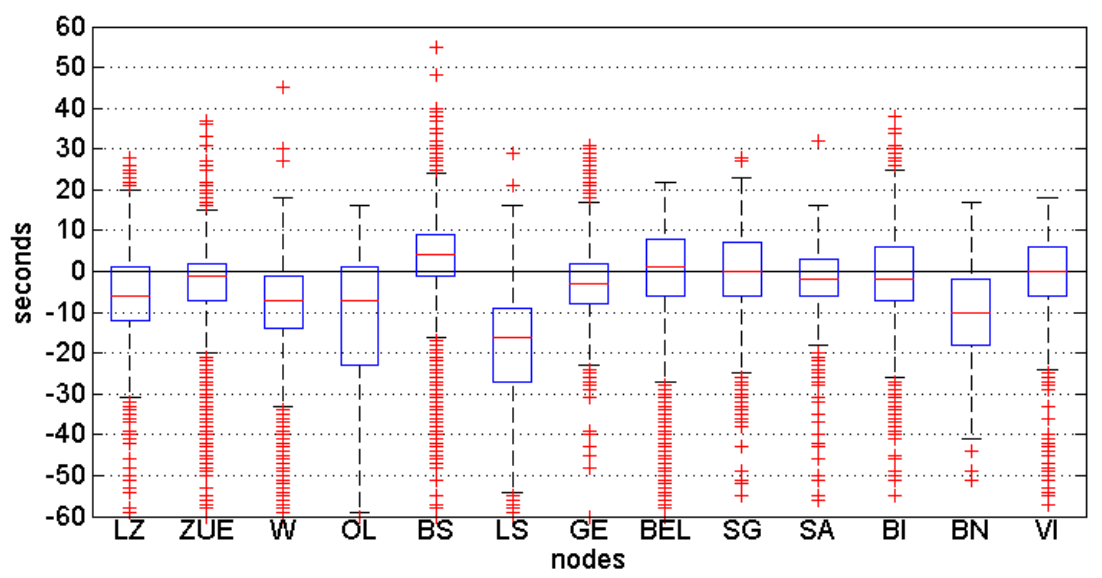

Figure 3: $\quad$ Boxplots of measurement errors in seconds.

\section{Ongoing timetable improvement}

This traffic data serves as a basis for continual improvements to the timetable. The stability of the timetable is regularly checked - on a daily basis when the timetable changes, and every month while the new timetable is being designed. For this purpose, the original version of Open Timetable (see Ullius [1] or Nash, Ullius [2]) has been completely rewritten in Java, the more complex calculations have been parallelised and many new functions have been introduced. At SBB, all the timetable schedulers have access to this system and are required to familiarise themselves with and take due account of the dynamics of the Swiss railway system when planning the timetable.

\subsection{Traffic data format}

The traffic data exported from the RCS system for analysis using the Open Timetable system contains the following elements:

train number, date of movement, time of arrival (date and hour, minute, second), time of departure (date and hour, minute, second), arrival delay (in seconds), departure delay (in seconds), name of the station, track and type of train.

The database contains traffic data on a 3-year rolling window for around 1,000 stations and 10,000 trains per day.

As can be seen, scheduled operations are not explicitly included in this data; they must be deduced from the arrival and departure times and their respective delays. It is therefore not uncommon to find different "scheduled" train paths for the same train. There may be several reasons for this: the train has been diverted, part of the train's journey has been cancelled, a train path has been altered for a single day, etc. In the Open Timetable system used to analyse this data, an algorithm selects for each train, from all the scheduled train paths, the longest train path that contains the most frequently recurring succession of stations. 


\subsection{Time-distance graph}

The most commonly used view is the time-distance graph, which visualises the movements of passenger trains on the lines. Plotting the data from Monday to Friday for an entire month gives the following result:

This kind of graph contains a great deal of information about the stability of the traffic. To start with, it can be seen that the general deterioration visible on the peak hour graph on the left diminishes on the graph on the right for all trains operating outside peak hours. The differing amounts of white space visible on these two graphs give a good indication of the system's capacity to absorb delays. Plotting all the daily movements forms a kind of footprint for each individual train. This footprint will vary as the train moves along its route, allowing us to identify the critical places. Once these critical places have been recognised, each timetable scheduler will evaluate and set the necessary margins, adapt the train paths to reduce instabilities or add new train paths in the most appropriate places to ensure optimum stability.
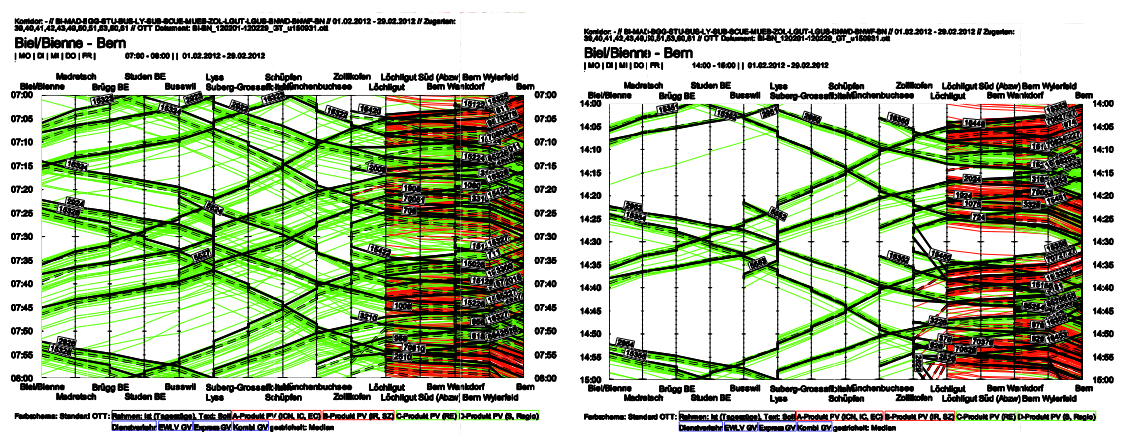

Figure 4: $\quad$ Time-distance graphs.

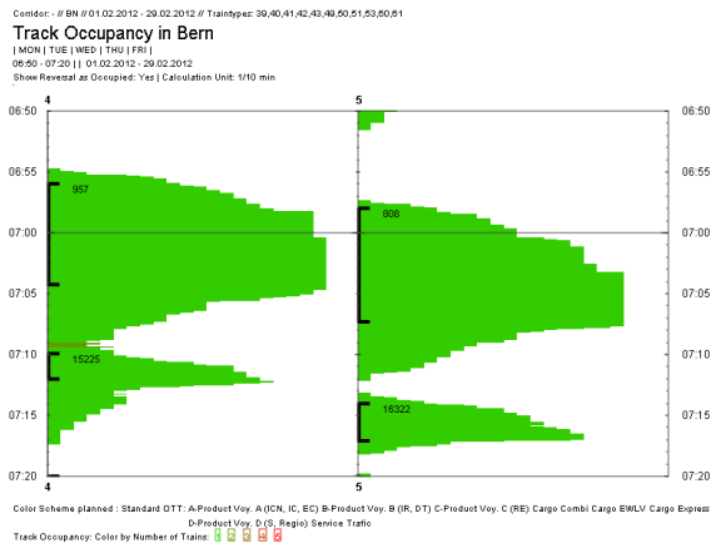

Figure 5: $\quad$ Occupancy of tracks 4 and 5, Berne, February 2012. 


\subsection{Track occupancy graph}

A specific graphical representation of track occupancy has been developed to help visualise the dynamics of rail traffic in stations:

For each track in the station, the time axis is divided into tenths of minutes and the number of occupancies for each segment is shown for all the days under consideration. It is thus possible to identify the times when real occupancies occur. This allows us to identify the trains and the critical movements in the stations so that we can decide, for example, on the feasibility of an additional stop. Areas may be coloured in to show three things: the occupancy rate, the type of train occupying the track, or, as here, the number of different trains occupying the track at the same time on different days.

\section{Statistical analysis}

For a long time now, statistical analyses at SBB have been made with the help of histograms. More recently, a new type of boxplot has been introduced which can better compare different sets of data.

\subsection{Analysis criteria available}

Using the basic data defined in point 3.1, different key criteria for constructing a timetable are calculated: arrival delay, departure delay, dwelling time, travel time, deviation from scheduled dwelling time and deviation from scheduled journey time. Some of these criteria can be derived directly from the data, while others have to be calculated by combining several types of data.

\subsection{Data sets}

One very efficient way of making it easier to identify the source of problems in statistical analyses is to group the data so as to highlight the differences between the sets. The data has the following three dimensions: time, place and train. On the basis of these three dimensions, the following sets have been integrated into the Open Timetable system: months, weeks, days, times, periods of the day (morning peak, off peak, evening peak, evening, night), stations, tracks in stations, types of train, train families in each direction and train numbers. The use of these sets means that when punctuality targets are not achieved in a station, it is possible to identify the set that is causing a problem and restrict field research to this particular set.

\subsection{Graphical representations}

The Open Timetable system allows statistical analyses to be presented in three ways: histograms, boxplots (adapted, see 4.3.2) and line graphs. These types of representation are available for all the criteria mentioned in 4.1 and for all the groups mentioned in 4.2 . 


\subsubsection{Histograms}

Representing data in the form of a histogram is very useful for visualising whether punctuality targets have been achieved. At SBB, the punctuality targets used for passenger train movements are defined by the percentage of trains (92.1\% in 2012) that must arrive at the 13 principal connection nodes with less than 3 minutes' delay. By colouring in the histogram, it is easy to identify the minute at which the $92.1 \%$ quantile of all arrivals is situated and thus measure the number of minutes by which the target is missed (in one direction or the other):

This kind of graph also permits a visual comparison of arrivals in different nodes. The examples below show Basel and Winterthur stations:

On the left-hand histogram, arrivals at the Basel node form a relatively wide histogram. By contrast, on the right-hand histogram, which depicts the Winterthur node, arrivals form a much more pointed histogram. However, this way of comparing histograms presents two problems: first, the data quantities are not always the same, which can easily falsify the analysis of the shapes and the conclusions drawn; and second, from a purely cognitive point of view, it is necessary to look for the differences because they are not immediately obvious. It should also be noted that the vertical scales are not the same in this example.

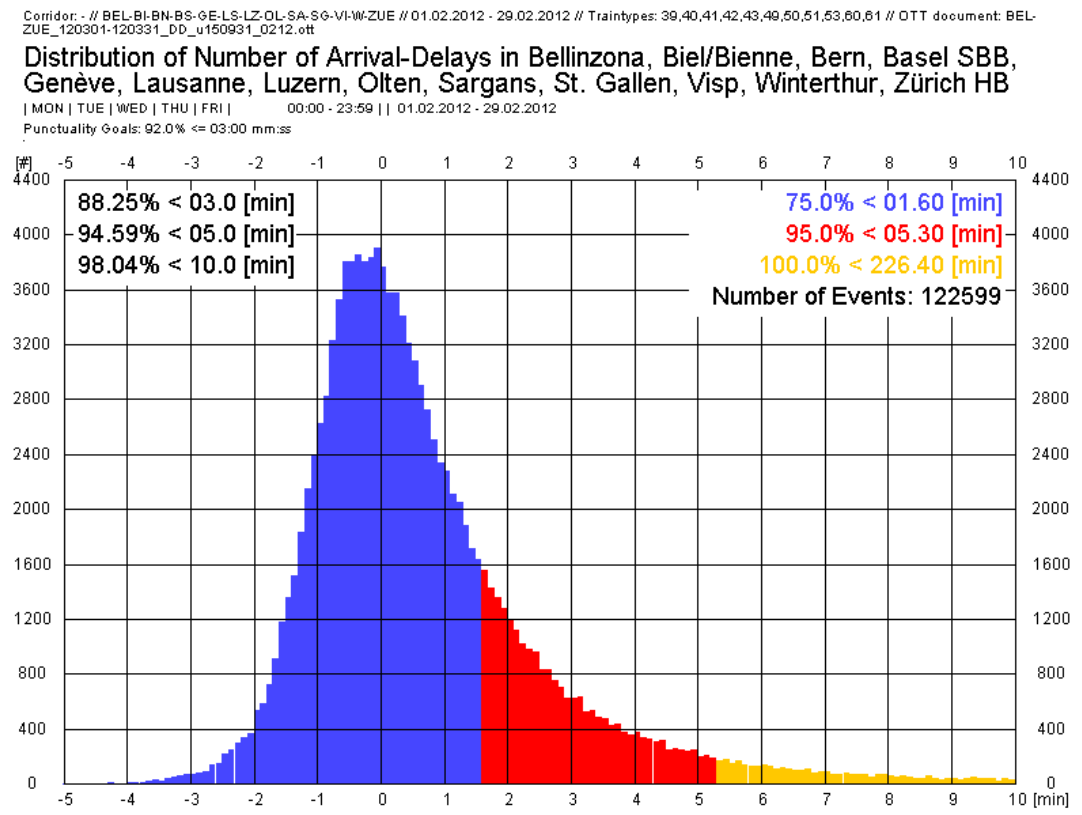

Figure 6: Histogram of arrivals in February 2012.

\subsubsection{Boxplots}

The great strength of boxplots is that they enable a large amount of data to be compared with "5-number summary". However, the original definition of the 
whiskers, by Tukey [3], is better adapted to normal probability densities. The whiskers, defined as 1.5 times the interquartile range, are a good rule of thumb to identify outliers but do not allow asymmetry in a probability density to be properly identified. Just like the data analysed in Goverde [4], arrivals and departures in Switzerland are not of the normal type and are strongly asymmetrical, as can be seen in Figure 7: Examples of histograms.
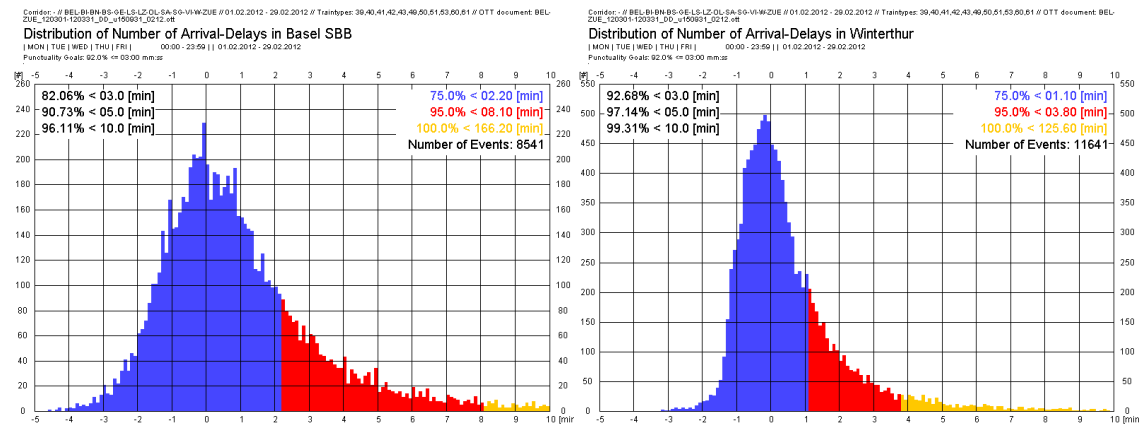

Figure 7: Examples of histograms.

As a result, an alternative definition of the whiskers was introduced in the Open Timetable system so that these asymmetries in the data could be visualised better:

Thanks to the use of the $5 \%$ and $95 \%$ quantiles, the shape of the probability density is represented much more clearly (see also point 5 , Top down analysis method).

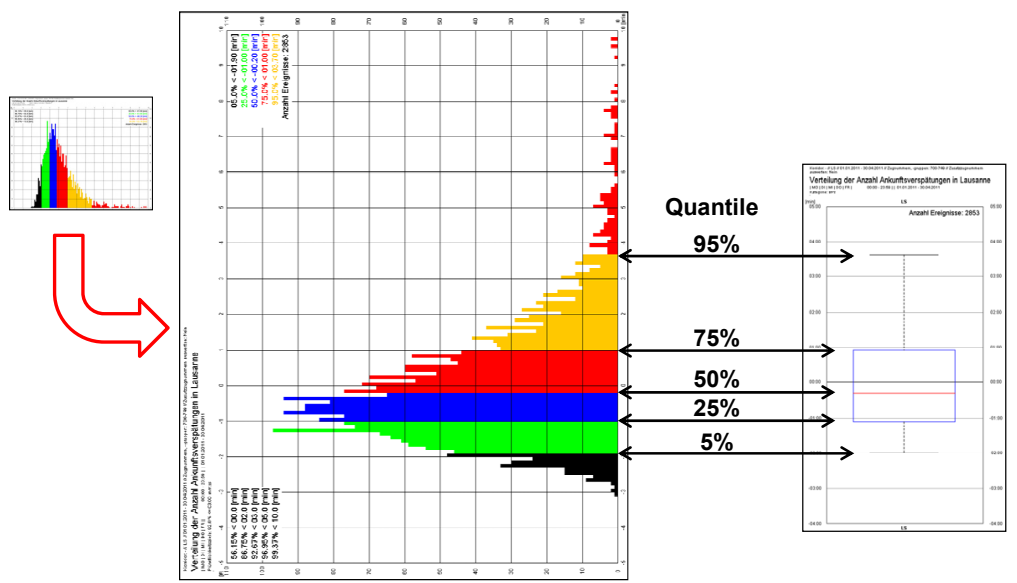

Figure 8: Alternative definition of boxplots. 


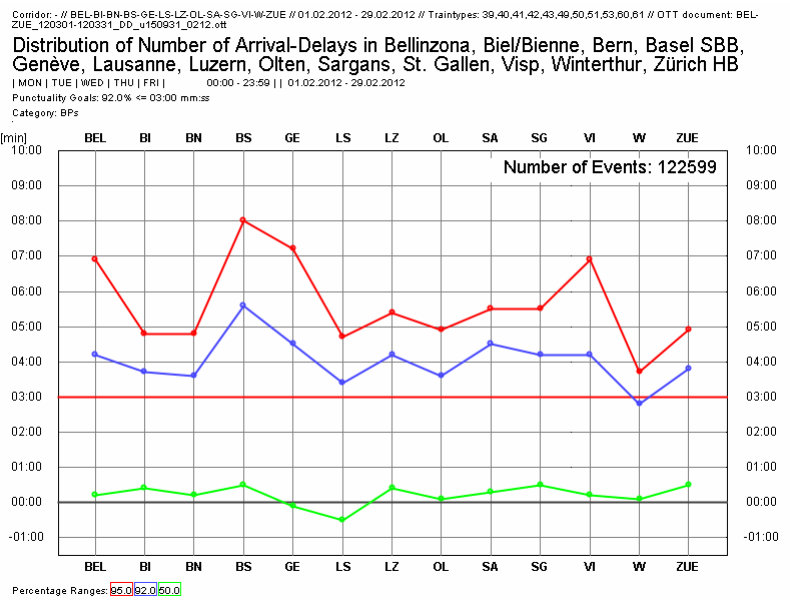

Figure 9: $\quad$ Line graphs for $95 \%, 92 \%$ and $50 \%$ quantiles.

\subsubsection{Line graphs}

In practice, boxplots are sometimes criticised for giving too much information to people who are not used to statistical tools. This is why a line graph of one or more quantiles can be used to get the main message across to a wider audience. The value of the quantile is calculated for each data set and these figures are linked to each other for the same quantile:

This graph contains less information than the one in Figure 10: Boxplots of arrivals in February 2012, but it is easier to read and still demonstrates the influence of certain groups of data.

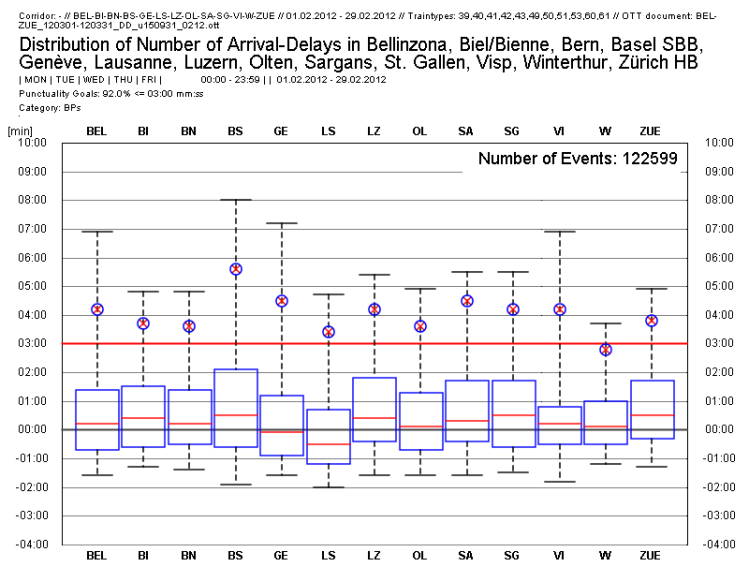

Figure 10: Boxplots of arrivals in February 2012. 


\section{Top down analysis method}

Throughout Switzerland in 2012, 92.1\% of trains should arrive with less than 3 minutes' delay in the 13 major stations. As can be seen in Figure 6: Histogram of arrivals in February 2012, this target was not achieved in February.

This kind of graph makes it difficult to determine where there is scope for improvement. In order to identify the points that need improvement, let us apply the station set to this data for all of Switzerland and visualise the results in the form of boxplots:
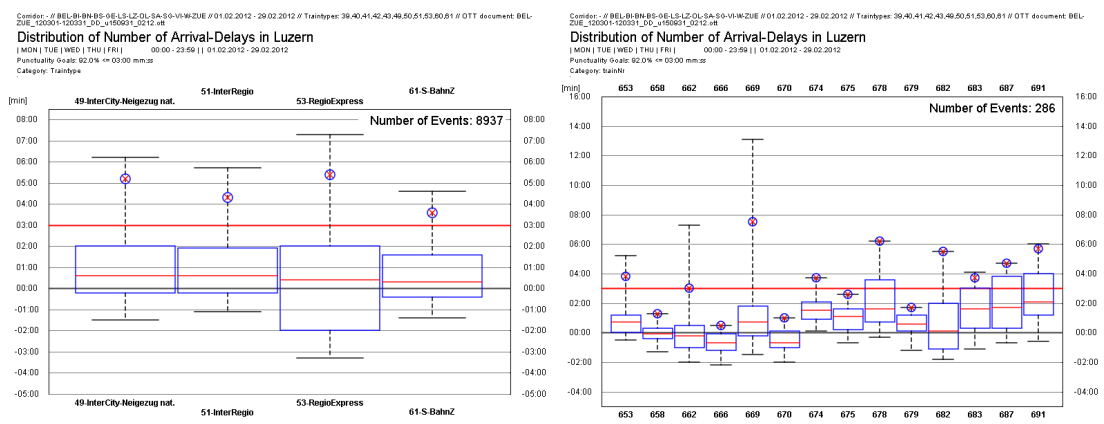

Figure 11: Boxplots with two data sets.

The boxplots show at a glance that Basel station (BS) has the greatest potential for improvement.

Once the nodes that are of interest have been identified, we can then focus on them. Although the next part of the example is based on Lucerne station (LZ), any station that has been identified can be investigated in a similar manner. By restricting ourselves to the data for a single station, we can use boxplots and the different data sets to identify which group of data is of interest:

On these boxplots, it can be seen on the left that domestic tilting trains (type 49) are among those that have most often failed to meet the 3-minute target. If we then look at the type 49 trains one by one, the right-hand graph is obtained. On this graph, trains $691,687,683$ and 678 can be identified as the ones which are late most often.

If these trains produce similar results over several months, a targeted investigation into the causes on the ground will allow specific action to be taken, the effects of which can then be monitored using similar graphs.

\section{Analysis of specific train runs}

If a particular train is shown to be involved in the poor performance of a station, analysing how it runs will enable the possible causes to be identified:

This graph shows that train 683 mostly runs ahead of time as far as Emmenbrücke station. It only starts to run late before entering Lucerne station. 
Having discovered this, the scheduler can now analyse the situation by considering all the trains at this place and can then try to eliminate conflicts by adapting the use of the routes and/or the timetables of the different trains involved.

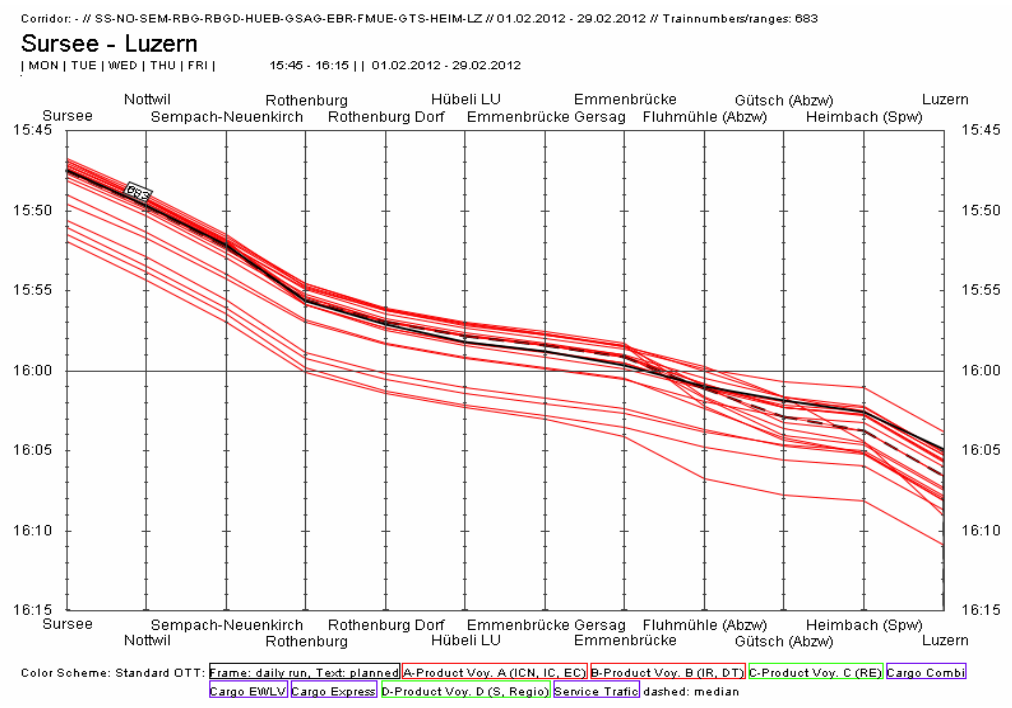

Figure 12: Train run: train 683.

\section{Conclusions and outlook}

This article has reviewed different ways of visualising traffic data by standard methods within the framework of ongoing timetable improvement. In particular, an alternative definition of the boxplot which is better adapted to rail traffic data has been introduced. A top down method to track down the biggest traffic problems in respect of punctuality targets has also been presented. These methods facilitate and greatly accelerate the identification of potential for improving a timetable that is in production and monitoring the effectiveness of the steps taken. However, the fundamental feasibility of a clockface timetable is only guaranteed when all train delays can be absorbed within a finite time. In cases where delays cannot be absorbed, there is a risk of knock-on delays - one train delays another which in turn delays another and so on - thus jeopardising the feasibility of the entire timetable. Methods have been devised for identifying pairs of trains which are in conflict: see for example Boris et al. [5] or Holger et al. [6]. However, these methods are only the first step towards an overview of knock-on delays. For each day under consideration, it will still be necessary to link up these train pairs and identify the absorption of the delays related to each train movement. On that basis, we can then aggregate these knock-on delays 
over one or more months in order to identify those that are most critical to timetable stability.

\section{References}

[1] Ullius, M., Verwendung von Eisenbahnbetriebsdaten für die Schwachstellen- und Risikoanalyse zur Verbesserung der Angebots- und Betriebsqualität, Ph.D. thesis, ETH Zurich, 2005.

[2] Nash, A. and Ullius, M. Optimizing railway timetables with OpenTimeTable, Computers in Railways IX, 637-646, 2004.

[3] Tukey, J. W., Exploratory Data Analysis, Addison-Wesley, 1977.

[4] Goverde, R., Punctuality of Railway Operations and Timetable Stability Analysis (Chapter 5), TRAIL, 2005.

[5] Boris, C., Goethals, B., Tassenoy, S, Verboven, S., Mining Train Delays, IDA, 113-124, 2011.

[6] Holger, F., Gelashvili, R., Graffagnino, G., Nunkesser, M, Mining Railway Delay Dependencies in Large-Scale Real-World Delay Data. Robust and Online Large-Scale Optimization, 354-368, 2009. 